\title{
Advanced Curation of Astromaterials for Planetary Science Over the Next Decade
}

Francis M. McCubbin ${ }^{1 *}$, Judith H. Allton ${ }^{1}$, Jessica J. Barnes ${ }^{2}$, Michael J. Calaway ${ }^{3}$, Catherine M. Corrigan $^{4}$, Justin Filiberto ${ }^{5}$, Marc. D. Fries ${ }^{1}$, Juliane Gross ${ }^{1,6}$, Andrea D. Harrington ${ }^{1}$, Christopher D. K. Herd ${ }^{7}$, Aurore Hutzler ${ }^{5}$, Hope A. Ishii ${ }^{8}$, Timothy J. McCoy ${ }^{4}$, Kevin McKeegan ${ }^{9}$, Julie L. Mitchell $^{1}$, Larry R. Nittler ${ }^{10}$, Aaron B. Regberg ${ }^{1}$, Kevin Righter ${ }^{1}$, Christopher J. Snead ${ }^{3}$, Rhonda Stroud $^{11}$, Kimberly T. Tait ${ }^{12}$, Toru Yada ${ }^{13}$, Ryan A. Zeigler ${ }^{1}$, Michael E. Zolensky ${ }^{1}$, Eileen K. Stansbery ${ }^{1}$

${ }^{1}$ NASA Johnson Space Center, Mailcode XI2, 2101 NASA Parkway, Houston, TX, 77058, USA

${ }^{2}$ Lunar and Planetary Laboratory, University of Arizona, 1629 E University Blvd, Tucson, AZ, 85721, USA

${ }^{3}$ Jacobs-JETS Contract, NASA Johnson Space Center, 2101 NASA Parkway, Houston, TX, 77058, USA

${ }^{4}$ Department of Mineral Sciences, National Museum of Natural History, Smithsonian Institution, Washington, DC, USA

${ }^{5}$ Lunar and Planetary Institute, USRA, 3600 Bay Area Boulevard, Houston, TX, 77058-1113, USA

${ }^{6}$ Rutgers University, Department of Earth and Planetary Sciences, Piscataway, NJ, 08854, USA

${ }^{7}$ Department of Earth and Atmospheric Sciences, 1-26 Earth Sciences Building, University of Alberta, Edmonton, AB, T6G 2E3, Canada

${ }^{8}$ Hawai'i Institute of Geophysics and Planetology, University of Hawai'i at Manoa, 1680 East-West Road, POST 602, Honolulu, HI, 96822, USA

${ }^{9}$ Department of Earth, Planetary, and Space Sciences, University of California, Los Angeles, CA, 90095, USA

${ }^{10}$ Earth and Planets Laboratory, Carnegie Institution of Washington, 5241 Broad Branch Road, Washington, DC, 20015, USA

${ }^{11}$ Materials Science and Technology Division, U.S. Naval Research Laboratory, Washington, DC, 20375, USA

${ }^{12}$ Department of Natural History, Royal Ontario Museum, 100 Queen's Park, Toronto, ON, M5S 2C6, Canada

${ }^{13}$ JAXA, 3 Chome-1-1 Yoshinodai, Chuo Ward, Sagamihara, 252-5210, Japan

*Primary author email address \& phone number: francis.m.mccubbin@nasa.gov; 281-483-5126 


\section{Executive Summary}

Advanced curation is a cross-disciplinary field of research and development aiming to improve curation and sample acquisition practices in existing astromaterials collections and to enable future sample return activities. The primary result of advanced curation is to both reduce and quantify contamination to astromaterials and preserve the scientific integrity of all samples from mission inception to scientific analysis. Over the next decade, NASA should support advanced curation research and monitoring efforts as they pertain to improving our current collections and preparing for samples from current and future astromaterials acquisition activities. We highlight here five advanced curation activities of critical importance for the success of sample science supported by NASA over the coming decade, including: 1) supporting efforts to build contamination knowledge collections as part of sample return missions, which requires curation involvement from the earliest stages of sample return mission planning; 2) supporting Earth-based astromaterials collection campaigns of meteorites and cosmic dust as they represent relatively inexpensive sample acquisition activities that continue to grow NASA's astromaterials collections and enable new discoveries; 3) preparing to curate and process samples under "cold" conditions to enable return of samples from volatile-rich Solar System targets like permanently shadowed regions on the lunar surface or comets; 4) determining how best to combine clean room technology and biosafety technology into one infrastructure to support curation of samples from bodies designated as Category V: Restricted Earth Return; and 5) supporting real-time monitoring and testing of curation labs to verify that sample processing environments remain clean from the standpoint of inorganic, organic, and biological contamination.

\section{Introduction}

Just as terrestrial samples record the natural history of our planet, astromaterials hold the natural history of our Solar System and beyond. Astromaterials acquisition and curation practices have direct consequences on the types of questions that can be answered about our Solar System and the degree of precision that can be expected of those answers. Advanced curation was developed as a cross-disciplinary field to improve acquisition and curation practices in existing astromaterials collections and for future sample return activities, including meteorite and cosmic dust samples that are collected on Earth. These goals are accomplished through research and development of new innovative technologies and techniques for sample collection, handling, processing, characterization, and analysis of astromaterials.

The initial curation of astromaterials began in earnest with the curation of meteorite samples in museums in 1748 at the Natural History Museum in Vienna [1]. Meteorite recovery and curation practices have evolved significantly since those initial efforts, and are highly dependent on many factors. These factors include the knowledge and resources of the finder and the financial and technical support available for the collection in which the sample is curated. The scientific importance of the sample can also be a determining factor, but this is predicated on the aforementioned factors. All meteorites, regardless of how they were handled from recovery to curation, have experienced uncontrolled entry and exposure to the terrestrial environment, including, at minimum, the terrestrial atmosphere and the ground. This exposure results in terrestrial contamination, the amount of which is dependent on the physicochemical properties of the meteorite, the conditions at the fall site, and the amount of exposure time to the terrestrial environment. Consideration of these factors can also be determining factors in how a meteorite sample is curated. An overview of meteorite collections, their contents, and curation practices is available in McCall et al. [2]. 
Until the 1960's, delivery of all astromaterials to Earth were unplanned events that required reactionary responses for recovery and curation. However, the initiation of the Apollo program made possible the direct return of pristine astromaterials from another body and established the need to design a facility to keep those samples in a pristine state for an extended period of time. Planning for the Lunar Receiving Laboratory (LRL) began in 1964, and the facility was completed in $1967[3,4]$. As part of this planning, stringent protocols in the handling, storage, and processing of samples were developed. These protocols ensured that portions of the samples remained pristine or as close to an "as returned" state as possible in perpetuity to enable future scientific discoveries from the returned samples.

The planning process for curation prior to the return of the Apollo 11 samples set the precedent that curation involvement and planning begins at the inception of a sample return mission; this founding principle has guided all sample return missions since Apollo [e.g., 5-7]. There have been a total of 12 successful sample return missions, including six crewed Apollo missions from NASA, three uncrewed Soviet lunar sample return missions, the NASA Genesis mission that returned solar wind collected from the Earth-Sun Lagrange point 1 (L1), the NASA Stardust mission that returned particles from the coma of Comet Wild 2 and from interstellar space, and JAXA's Hayabusa mission that returned material from the surface of asteroid Itokawa (Table 1). In addition, there are two sample return missions in flight: JAXA's Hayabusa2 mission that will return samples from the asteroid Ryugu and NASA's OSIRIS-REx mission that will return material from the asteroid Bennu (Table 1) in the coming decade. With each successive sample

\begin{tabular}{|l|l|l|l|}
\hline \multicolumn{4}{|l|}{ Table 1. Planetary Sample Return Missions } \\
\hline Program/Mission & Returned to Earth & Destination & Returned Sample \\
\hline Apollo 11 (USA, NASA) & July 24, 1969 & Moon: Mare Tranquillitatis & $21.55 \mathrm{~kg}$ \\
\hline Apollo 12 (USA, NASA) & November 24, 1969 & Moon: Oceanus Procellarum & $34.30 \mathrm{~kg}$ \\
\hline Luna 16 (USSR) & September 24, 1970 & Moon: Mare Fecunditatis & $101 \mathrm{~g}$ \\
\hline Apollo 14 (USA, NASA) & February 9, 1971 & Moon: Fra Mauro Highlands & $42.80 \mathrm{~kg}$ \\
\hline Apollo 15 (USA, NASA) & August 7, 1971 & Moon: Hadley-Apennine & $76.70 \mathrm{~kg}$ \\
\hline Luna 20 (USSR) & February 25, 1972 & Moon: Apollonius Highlands & $55 \mathrm{~g}$ \\
\hline Apollo 16 (USA, NASA) & April 27, 1972 & Moon: Descartes Highlands & $95.20 \mathrm{~kg}$ \\
\hline Apollo 17 (USA, NASA) & December 19, 1972 & Moon: Taurus-Littrow & $110.40 \mathrm{~kg}$ \\
\hline Luna 24 (USSR) & August 22, 1976 & Moon: Mare Crisium & $170.1 \mathrm{~g}$ \\
\hline Genesis (USA, NASA) & September 8, 2004 & Earth-Sun Lagrange 1 & $\begin{array}{l}\text { Implanted Solar } \\
\text { Wind Atoms }\end{array}$ \\
\hline Stardust (USA, NASA) & January 15, 2006 & Comet Wild 2 / Interstellar & $\begin{array}{l}\text { Small Particles } \\
\text { Captured in } \\
\text { Aerogel and Al } \\
\text { foils }\end{array}$ \\
\hline Hayabusa (Japan, JAXA) & June 13, 2010 & Asteroid 25143 Itokawa & $\begin{array}{l}\text { Tens of } \\
\text { thousands of } \\
\text { recovered small } \\
\text { particles }\end{array}$ \\
\hline Hayabusa2 (Japan, JAXA) & $\begin{array}{l}\text { December 2020 } \\
\text { (Planned; in Flight) }\end{array}$ & Asteroid 162173 Ryugu & $\begin{array}{l}\text { 0.1 to 10 g } \\
\text { Planned }\end{array}$ \\
\hline OSIRIS-REx (USA, NASA) & $\begin{array}{l}\text { September 2023 } \\
\text { (Planned; in Flight) }\end{array}$ & Asteroid 101955 Bennu & $\begin{array}{l}\text { 0.06 - } 2 \mathrm{~kg} \\
\text { Planned }\end{array}$ \\
\hline
\end{tabular}

return mission, an important set of lessons are learned which inform subsequent sample return missions, including curation standards and practices. Building upon Apollo and later sample return 
missions, a series of lessons learned and best practices for future sample return missions have been developed and are part of advanced curation [7].

Lessons learned not only inform our best practices, but they also help to identify strategic knowledge gaps that require new research to fill. Furthermore, if we look only at improving upon our current curation capabilities, we will not be prepared when returned samples require care that is very different from those within our current collections. At present, most returned samples are micro-to-macroscopic particles, with the exception of the Genesis solar wind atoms that are implanted within a number of high purity material substrates. Most of the samples are kept close to room temperature and, when kept in the pristine environments of a clean laboratory, will maintain their fidelity indefinitely. However, future sample return missions could bring back samples that require storage and handling conditions outside of current capabilities, including gases, liquids, ices, or biological materials. Successfully curating these environmentally sensitive materials will require new research and capabilities that are being developed in the field of research known as advanced curation. Consequently, advanced curation will help facilitate the return of new sample types that will enable answering new high-priority science questions.

Advanced curation has two primary goals: 1) expansion of the sample handling, processing, and storage capabilities of astromaterials facilities to prepare for future sample return missions and Earth-based collection of astromaterials, and 2) maximization of the science returns of existing astromaterials sample collections. In addition, advanced curation includes testing and evaluation of new technologies and operational procedures for future sample return missions through human and robotic analog studies. In this contribution, we discuss four broad topics in advanced curation that, over the next decade, are critical to improving sample acquisition and curation practices. These topics include: 1) monitoring, testing, and developing new curation infrastructure for inorganic, organic, and biological contamination; 2) development of new storage, processing, and sample handling capabilities for future sample return missions; 3) advancements and improvements in astromaterials acquisition capabilities on Earth (i.e., the collection of meteorites and cosmic dust); and 4) development of contamination knowledge (CK) strategies for maximizing the science returns of sample-return missions. The primary result of advanced curation research is to both reduce and quantify contamination of astromaterials and preserve the scientific integrity of all samples from mission inception to secure delivery of samples to Earth-based laboratories for in-depth scientific analysis. Advanced curation serves as an important scienceenabling activity, and the collective lessons learned from previous spacecraft missions and the results of advanced curation research will work in tandem to feed forward into better spacecraft designs and enable more stringent requirements for future sample return missions and Earth-based sample acquisition. The specific focus of this whitepaper is to communicate the need for advanced curation activities to support and enable return of new sample types and to maximize science returns on all astromaterials. Related whitepapers that address the scientific value of existing astromaterials collections [8], new sample return targets [e.g., 9-13], and Earth-based extraterrestrial sample collection [14] have also been submitted.

\section{Monitoring, testing, and developing curation infrastructure}

All NASA sample return curation facilities are designed and built to meet specific controlled environment and cleanliness standards for the curated samples. Curation infrastructure is defined as the engineering systems that control the sample's storage and processing environment. This definition incorporates brick and mortar, temporary, modular, and mobile facilities. In addition, specialized equipment is included such as isolation chambers, gloveboxes, and desiccators that have the ability to alter the atmospheric chemistry, temperature, and pressure 
of the environment. Curation infrastructure is derived from many industries including the nuclear, biotechnology, pharmaceutical, and semiconductor industries. Methods and techniques are either borrowed, augmented, or invented to maintain the controlled environment necessary to mitigate terrestrial cross-contamination. Contamination covers any element that could compromise sample integrity. To quote the definition from Dworkin et al. [15], "pristine" means that "no foreign material is introduced to the sample in an amount that hampers the ability to analyze the chemistry and mineralogy of the sample." While sample return missions generally designate contamination limits on specific elements and compounds at time of launch on the basis of focused science goals, samples are allocated to researchers over time to study effectively everything on the periodic table. Therefore, the implementation of curation infrastructure should be mindful that anything could be a contaminant to some research group. Modern cleanroom facilities have substantial infrastructure footprints that require continual monitoring to ensure they operate within the defined strict contamination control guidelines. Staying within these guidelines requires continuous monitoring and testing of the labs to verify that the sample processing environments remain clean from the standpoint of inorganic, organic, and biological contamination. As it is unrealistic to eliminate all contamination, careful monitoring and CK must be conducted. To this end, curation laboratories that house astromaterials must develop and continue to update numerous protocols and methods to monitor cleanliness. As sample return missions commence in the next decade, advanced curation will continue to explore and develop new types of curation infrastructure that include the use of new construction materials, isolation containment technologies, cleanroom technologies and biocontainment strategies to meet the increasing demand for more stringent science requirements.

\section{Development of new storage, processing, and sample handling capabilities}

As technological advancements and new ideas expand the variety and scope of scientific questions that can be answered with astromaterials samples, so expands the need for better storage, processing and sample handling capabilities of curation laboratories that house and process astromaterials samples. Over the coming decade, we will need to improve our ability to curate and process samples under "cold" conditions (at or below the freezing point of water), develop remote robotic handling of samples in isolators without gloves, and improve our small particle micromanipulation systems.

The ever-expanding plans for the return of samples from volatile-rich Solar System targets and/or targets of astrobiological significance (e.g., volatile-rich samples from the lunar poles, comet nucleus samples, icy moons, Mars, and other samples of biological importance) necessitates the development of curation at temperatures below that of typical curation facilities $\left(20{ }^{\circ} \mathrm{C}\right)$. Temperature requirements depend primarily on which volatiles are expected within the returned sample, which in turn relate to the conditions under which the material formed and has since been preserved. The term "cryogenic" is defined as relating to temperatures below $-183{ }^{\circ} \mathrm{C}$; the normal boiling points of the noble gases, oxygen, nitrogen, and air lie below this temperature. More generally, "cryogenic" refers to temperatures below approximately $-150{ }^{\circ} \mathrm{C}$. The term "hightemperature cryogenic" is used to refer to temperatures from the boiling point of liquid nitrogen, $196{ }^{\circ} \mathrm{C}$, up to $-50{ }^{\circ} \mathrm{C}$, the generally defined limit of cryogenics [e.g., 16]. The curatorial temperatures for terrestrial materials, including tissue samples and ice cores, include: $\leq-20{ }^{\circ} \mathrm{C}$ (the temperature of typical walk-in freezers in which physical processing and documentation takes place); $\leq-40{ }^{\circ} \mathrm{C}$ for archival storage (e.g., of ice cores); and -80 to $-196{ }^{\circ} \mathrm{C}$ (liquid nitrogen) for biological samples [e.g., 17-18]. Thus, with the exception of biological tissue storage, the field of Earth materials curation has not yet entered the realm of cryogenics, although cold curation and cold processing of astromaterials are in development [e.g., 19-20]. 
In addition to cold curation practices, the scientific community has identified Mars Sample Return (MSR) as a high priority sample return activity in the coming decade, and current efforts relating to MSR are focused on a multi-mission campaign, the first of which is the Mars 2020 rover mission to Jezero Crater. MSR will yield many unique challenges for curation that must be addressed in the time period being considered by the present decadal survey. Astromaterials curation facilities have already been asked to curate biological materials sampled from flight hardware for the Mars2020 mission to include in a CK collection. Although there are no requirements that the martian samples be kept cold, biological sampling of the flight hardware includes swab samples, liquids, isolated pure cultures of bacteria and fungi, and DNA samples, which will need to be kept under cold conditions. Furthermore, processing of returned martian samples will require advancements in remote robotic handling in isolators without gloves, including the use of small particle micromanipulation systems.

Finally, NASA Policy Directive (NPD) 8020.7G, Biological Contamination Control for Outbound and Inbound Planetary Spacecraft, complies with the UN Space Treaty and COSPAR planetary protection policy stating "the Earth must be protected from the potential hazard posed by extraterrestrial matter carried by a spacecraft returning from another planet or other extraterrestrial sources." NASA Procedural Requirements (NPR) 8020.12D, Planetary Protection Provisions for Robotic Extraterrestrial Missions, outlines requirements for meeting the NPD 8020.7G and specifies planning documents and reviews for Category V Restricted Earth Returns. The Planetary Protection Office classifies any "samples from Solar System bodies that may harbor indigenous life" as Category V: Restricted Earth Return, and Mars is designated as Class V Restricted Earth Return. Consequently, samples may require biosafety level 4 containment, which requires negative pressure labs to keep martian material contained. However, the samples must also remain pristine in order to maximize science returns, which typically requires storage and processing within positive-pressure clean-room facilities. The integration of these competing designs is required for MSR to be successful from a sample-science perspective, and considerable efforts will be needed to accomplish these goals and engineering challenges.

\section{Development of new astromaterials acquisition capabilities on Earth}

The study of astromaterials in the laboratory allows direct analysis of material arising from the full breadth of the history of our Solar System. The continuing advance of technology has improved not only our measurements but also our ability to minimize contamination and sample modification during collection of freshly fallen meteorites and cosmic dust. Some of these improvements are related to educating the public on proper methods of handling meteorites, but many of the technological advances have focused on improving our ability to track and find materials. In many cases, these improvements result in reduction of exposure time to uncontrolled conditions, which could reduce terrestrial contamination, especially if clean-collection practices are used during recovery operations. Collection of material has been steadily improved by advances in ground-based and satellite sensors, dissemination of information with the growth of the internet and various social media platforms, and especially by the flow of data through freely available data sources. Although the collection of astromaterials may seem, at first, to be a prerequisite of curation, one of the primary goals of advanced curation is aimed at maximizing the science returns of astromaterials samples, and improvements in sample collection techniques has a direct benefit to science. We are in the midst of an exciting period of growth of truly innovative astromaterials sample collection techniques on Earth, and continued support of these Earth-based collection campaigns in the coming decade is crucial. 


\section{Importance of contamination knowledge strategies for sample return missions to maximize science returns from samples}

The scientific value of the returned samples for all previous sample return missions have benefitted from maintaining an archive of CK materials including: 1) spacecraft hardware, spares, and flight-like coupons, 2) materials used in the fabrication of spacecraft hardware or construction of a curation lab, and 3) witness materials deployed during assembly, testing, and launch operations (ATLO) or during construction of the curation lab. CK is distinct from contamination control (verification of level 1 requirements) and from archiving hardware for future troubleshooting (engineering knowledge) because CK is for the scientific community and part of an astromaterials sample collection (Figure 1). The information gained from studying the collected reference materials and witness plates is defined as the $\mathrm{CK}$ of a sample collection, and the CK is crucial for verifying and validating scientific results. As part of a sample return mission, these CK samples are archived along with the returned samples and the CK samples are made available for allocation and analysis by the scientific stakeholders of a sample collection. These flown, flightlike, and non-flight reference materials and witness plates provide the scientific community investigating astromaterials with the fundamental ability to reconstruct the contamination history of a sample collection. Furthermore, they serve as a baseline from which to compare tantalizing results attained from the analysis of astromaterials. CK collections are a requirement for sample return missions because contamination control efforts cannot anticipate all possible contamination vectors that can occur during a dynamic sample return mission. In fact, all sample return missions have incurred non-nominal contamination to some degree, and in every case, the CK samples have helped to document these unplanned contamination events, improving and, in some cases, enabling scientific results from the returned astromaterials [e.g., 21-22]. It is crucial that NASA continues to support efforts to build $\mathrm{CK}$ collections as part of sample return missions, which requires curation involvement at the earliest stages of mission planning.

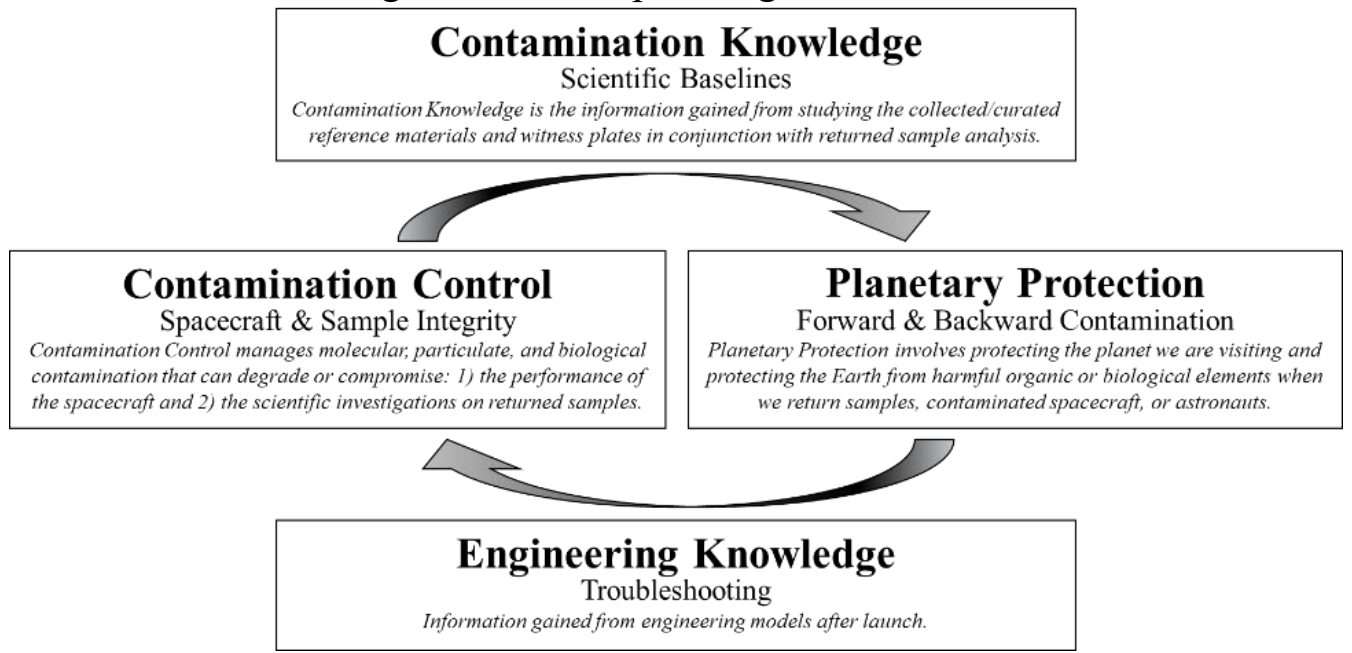

Figure 1. Cartoon illustrating the categories of samples needed for testing and verification of spaceflight missions. Each type of sample serves a different purpose and hence requirements for each sample collection related to these categories must be defined.

\section{Looking forward}

Advanced curation is critical to the success of sample return missions and Earth-based sample acquisition. It plays an integral part in enabling the high precision measurements that are often done on astromaterials samples to address big-picture scientific questions. Looking forward 
over the period encompassed by the decadal survey, advanced curation must prepare for sample return missions from any celestial source within the Solar System, including planets, moons, asteroids, comets, and/or cosmic dust. The direction and scope of advanced curation research is driven by: 1) existing strategic knowledge gaps identified through lessons learned from previous sample return missions and Earth-based programs that collect astromaterials; 2) the emerging needs of the scientific community that study astromaterials samples; and 3) the selection of new targets for sample return missions and the associated curation and sample handling requirements of those missions [e.g., 23-26]. The primary result of advanced curation is to both reduce and quantify contamination to astromaterials and preserve the scientific integrity of all samples from mission inception and through ATLO, sample collection, curation/preliminary examination on Earth, curation/storage, and allocation of samples to the scientific community for in-depth scientific analysis. Advanced curation is an interdisciplinary field of research and development and also serves as an important science-enabling activity. The collective lessons learned from previous spacecraft missions and the results of advanced curation research will work in tandem to feed forward into better spacecraft designs and enable more targeted science and engineering requirements for future sample return missions, which will enable new high-priority science questions to be answered.

References: [1] Brandstätter (2006) Geological Society, London, Special Publications 256, 123 133. [2] McCall et al. (2006) Geological Society, London, Special Publications, 256, 1-13. [3] Calaway et al. (2017) 48th LPSC, Abstract \#1224. [4] McLane et al. (1967) Science, 155, 525529. [5] Allen et al. (2011) Chemie der Erde - Geochemistry, 71, 1-20. [6] Yada et al. (2014) Meteoritics \& Planetary Science, 49, 135-153. [7] McCubbin et al. (2019) Space Science Reviews, 215, 48 [8] Stroud et al. (2020) White paper for Planetary Science and Astrobiology Decadal Survey 2023-2032. [9] McSween et al. (2020) White paper for Planetary Science and Astrobiology Decadal Survey 2023-2032. [10] Vander Kaaden et al. (2020) White paper for Planetary Science and Astrobiology Decadal Survey 2023-2032. [11] Westphal et al. (2020) White paper for Planetary Science and Astrobiology Decadal Survey 2023-2032. [12] Milam et al. (2020) White paper for Planetary Science and Astrobiology Decadal Survey 2023-2032. [13] Neveu et al. (2020) White paper for Planetary Science and Astrobiology Decadal Survey 2023-2032. [14] Ishii et al. (2020) White paper for Planetary Science and Astrobiology Decadal Survey 2023-2032. [15] Dworkin et al. (2018) Space Science Reviews, 214, 53. [16] Zohuri (2018) Physics of Cryogenics: An Ultralow Temperature Phenomenon. Elsevier. [17] Anchordoquy \& Molina (2007) Cell Preserv. Technol. 5, 180-188. [18] Rissanen et al. (2010) Applied Microbiology and Biotechnology, 88, 977-984. [19] Herd et al. (2016) Meteoritics \& Planetary Science, 51, 499-519. [20] Mitchell et al. (2018) 49th LPSC, Abstract \#1019. [21] Elsila et al. (2009) Meteoritics \& Planetary Science, 44, 1323-1330. [22] Day et al. (2018) Meteoritics \& Planetary Science, 53, 1283-1291. [23] Beaty et al. (2019) Meteoritics \& Planetary Science, 54, S1, S3-S152. [24] Haltigin et al. (2018) Astrobiology, 18, S1. [25] McLennan et al. (2011) Astrobiology, 12, 175230. [26] Vander Kaaden et al. (2019) Space Science Reviews, 215, 49. 